\title{
Análisis de indicadores técnicos que discriminan equipos ganadores y perdedores en el nuevo baloncesto Brasil
}

\author{
Analysis of performance indicators that discriminate \\ winning and losing teams in new basketball Brazil
}

\section{Análise dos indicadores técnicos que discriminam equipes vencedoras e perdedoras no novo basquete Brasil}

\author{
Prochnow, R.A. ${ }^{1}$, Reale, V.M.C. ${ }^{1}$, Santos, Y.Y.S. ${ }^{1}$, Monezi, L.A. ${ }^{1}$ e Mercadante, L.A. ${ }^{1}$
}

${ }^{1}$ Laboratório de Biomecânica e Instrumentação da Faculdade de Ciências Aplicadas da Universidade Estadual de Campinas - Limeira

Resumen: El objetivo de este estudio fue verificar cuales son los indicadores técnicos que diferencian a los equipos ganadores de perdedores en el baloncesto de Brasil Nueva (NBB) y en cada una de las siete temporadas analizadas. El objetivo específico fue verificar en el análisis discriminante el efecto de la normalización de los indicadores técnicos por el número de ataques. La muestra fue de 1912 partidos de temporadas entre 2008 y 2015 de la NBB. La tasa de éxito de la función discriminante fue de cerca de $82 \%$, sin diferencia sustancial entre el análisis con los indicadores técnicos normalizados y no normalizados. Los indicadores que discriminan el equipo ganador del perdedor en todos los juegos fueron los rebotes defensivos y asistencias. No se encontraron otros indicadores relacionados con el rodaje de dos y tres puntos en las diferentes estaciones.

Palabras clave: análisis discriminante; explorador; la eficiencia en el baloncesto

Abstract: The aim of this study was to verify which are the technical indicators that differentiate the winning teams from losers in the New Basketball Brazil (NBB) and in each of the seven seasons analysed. The specific objective was to verify in the discriminant analysis the effect of normalizing the technical indicators by the number of attacks. The sample consisted of 1912 matches of seasons between 2008 and 2015 of the NBB. The successful rate of the discriminant function was closely to $82 \%$, without substantial difference between the analysis with the normalized and non-normalized technical indicators. The indicators that discriminate the winner team from loser in were defensive rebounds and assists. Other indicators related to the two and three points shoot were found in different seasons.

Keywords: discriminant analysis; scout; efficiency in basketball

Resumo: O objetivo deste estudo foi verificar quais são os indicadores técnicos que diferenciam as equipes vencedoras de perdedores no Novo Basquete Brasil (NBB), e em cada uma das sete temporadas analisadas. O objetivo específico foi verificar na análise discriminante o efeito de normalizar os indicadores técnicos pelo número de ataques. A amostra foi composta por 1912 jogos de temporadas entre 2008 e 2015, da NBB. A taxa de sucesso da funçáo discriminante foi perto de $82 \%$, sem diferença substancial entre a análise com os indicadores técnicos normalizados e não normalizados. Os indicadores que discriminam as equipes vencedoras das perdedoras foram os rebotes defensivos e as assistências. Outros indicadores relacionados com os arremessos de dois e três pontos foram encontrados em diferentes temporadas.

Palavras chave: análise discriminante; estatística de jogo; eficiência no basquetebol

\section{Introdução}

Como um jogo desportivo coletivo, o basquetebol possui características de cooperaçáo e oposição, envolvendo açóes sucessivas de ataque e defesa, alternadas entre as equipes (Moreno, 1998). O resultado do jogo é dado pelo acúmulo de pontos obtidos nos ataques, e a vitória ou derrota das equipes está relacionada aos acertos e erros das açóes realizadas para conseguir a cesta (Galatti, Paes, Machado \& Seoane, 2015).

Diversos são os fatores que podem estar envolvidos nas vitórias e derrotas das equipes. Para compreender estes fatores, a análise estatística de desempenho vem se tornando

Dirección para correspondencia [Correspodence address]: Vitor de Magalhães Chaves Reale. E-mail: vitorm.chaves@hotmail.com uma metodologia largamente usada, trazendo um histórico da eficiência dos indicadores técnicos dessas equipes em seus jogos.

Em particular, dados quantitativos sobre o desempenho dos atletas ou das equipes durante as competiçóes são informações essenciais para as decisóes tomadas por uma comissão técnica, no decorrer da partida, nas fases de planejamento e treinamento, ou mesmo em pesquisas científicas ligadas ao esporte (Barros, Misuta, Menezes, Figueroa, Moura, Cunha, Anido \& Leite, 2002, Romarís, Refoyo \& Lorenzo, 2016).

Dentre os indicadores técnicos mais utilizados em estudos sobre basquetebol estáo os lances livres certos e errados, arremessos de dois e três pontos certos e errados, rebotes ofensivos e defensivos, assistências, bolas roubadas, erros, 
faltas e bloqueios de arremesso (Goméz, Lorenzo \& Barakat 2008a; Goméz, Lorenzo, Sampaio, Ibáñez \& Ortega, 2008b; García, Ibáñez, Santos, Leite \& Sampaio, 2013;), que são as variáveis fornecidas pelos sites oficiais das organizaçóes dos principais campeonatos de basquetebol de alto rendimento.

Estudos relatam a importância em analisar os indicadores técnicos que diferenciam as equipes vencedoras das perdedoras em diferentes contextos, como em jogos dentro e fora de casa, jogos equilibrados e desequilibrados, e em diferentes fases do campeonato (Trninić, Dizdar \& Lukšić, 2002; Sampaio \& Janeira, 2003; Gómez et al. 2008b; Csataljay, James, Hughes \& Dancs, 2012; García, Ibáñez, Gómez \& Sampaio, 2014; Puente, Coso, Salinero \& Abián-Vicén, 2015). Para identificar as variáveis que melhor discriminam as equipes vencedoras das perdedoras, é comumente usada a Análise Discriminante de Fischer (Ibáñez, Feu, Lorenzo, Gómez \& Ortega, 2008; García, Sáez, Ibáñez, Parejo \& Cañadas, 2013; García et al., 2014; Ibáñez, García, Feu, Lorenzo \& Sampaio, 2009). Estes trabalhos também realizam a normalização dos indicadores técnicos pelo número de ataques das equipes, (Oliver, 2004).

Ibáñez et al (2008), analisaram 807 jogos da temporada regular da segunda divisão da Liga Espanhola de Basquete (LEB1), e apontaram como indicadores técnicos discriminantes as assistências, bolas roubadas e tocos. Posteriormente, utilizando a amostra do trabalho anterior e incluindo 223 jogos do campeonato sub-20 da Liga Espanhola, (Ibáñez et al., 2009) apontaram como indicadores discriminantes os arremessos de dois pontos certos, rebotes defensivos e assistências.

Já García et al. (2013), analisaram 306 jogos da fase regular Liga Profissional Espanhola (ACB), e encontraram como indicadores técnicos discriminantes as assistências, os rebotes defensivos e os arremessos de dois e três pontos certos, porém, em 17 jogos das finais somente os rebotes defensivos. Em outro trabalho, García et al. (2014), apontaram os indicadores técnicos bolas roubadas e assistências como discriminantes em jogos balanceados, e assistências e arremessos de três pontos certos em jogos náo balanceados.

No Brasil, o basquetebol profissional começa a se reorganizar em 2008, com o surgimento do campeonato Novo Basquete Brasil (NBB), principal campeonato masculino adulto do país, organizado pela Liga Nacional de Basquetebol (LNB), criada em dezembro de 2008. A LNB disponibiliza as estatísticas oficiais de todos os jogos em seu site. São fornecidos dados de ambas as equipes e todos os jogadores, com suas posiçóes em quadra no momento das açôes. Poucos artigos foram publicados sobre jogos do NBB, a partir de dados fornecidos pela LNB (Carvalho \& Folle, 2014; Meneses, Gois \& Almeida, 2016; Reis \& Sampaio, 2014; Almas, 2015). Contudo, nenhum destes artigos analisou os indicadores téc- nicos nas condições de vencedores e perdedores e em função das diferentes temporadas.

O objetivo principal do presente estudo foi verificar quais os indicadores técnicos que diferenciam as equipes vencedoras das perdedoras, em todos os jogos do NBB e ao longo das sete temporadas. $\mathrm{O}$ objetivo específico foi verificar o efeito da normalização pelo número de ataques na análise discriminante dos indicadores técnicos.

\section{Método}

A amostra foi composta por 1912 jogos de sete temporadas do NBB, entre 2008 e 2015. Os dados dos indicadores técnicos das equipes em cada jogo foram retirados das estatísticas oficiais da LNB e as análises foram realizadas em ambiente Matlab: Os indicadores técnicos utilizados para as análises foram: arremessos de dois e três pontos certos e errados, lances livres certos e errados, rebotes defensivos e ofensivos, assistências, bolas roubadas, tocos e erros, que são os indicadores mais usados em pesquisas científicas. As faltas foram excluídas destas análises, pois não foram contabilizadas em todas as temporadas.

A obtenção dos dados a partir das tabelas da LNB foi feita manualmente, transferindo informaçôes entre ambientes computacionais diferentes, portanto, este processo é passível de erros. Neste sentido, para verificar a confiabilidade dos dados, foi construído um protocolo de procedimentos, utilizando gráficos do tipo boxplot, para identificação e correção de valores que representassem possíveis erros em cada indicador técnico.

Para identificar as variáveis que melhor discriminam as equipes vencedoras das perdedoras, utilizamos a Análise Discriminante de Fischer (García, et al 2013; Gómez et al., 2008b; Gómez, Lorenzo \& Barakat, 2008a; Ibáñez et al., 2009). A Análise Discriminante é uma formulação matemática de otimização para análises comparativas entre conjuntos de dados, que fornece valores de Coeficiente Estrutural (CE) para cada indicador técnico. Segundo Tabachnick e Fidell (2001), caso o coeficiente SC apresente valor absoluto igual ou maior que 0.30 , indica que este indicador discrimina os dois conjuntos de dados. Também foi realizada uma validação cruzada do modelo obtido pela análise discriminante, e calculada a taxa de acerto (Norušis, 2004).

Um dos processos comumente aplicado nos indicadores técnicos de jogos de basquetebol é a normalização de acordo com o número de ataques (Oliver, 2004), a fim de retirar a influência do ritmo de jogo (García et al., 2009; Ibáñez et al., 2008; Sampaio \& Janeira, 2003). A Análise Discriminante foi aplicada em dois conjuntos dos indicadores técnicos, para comparaçóes: dados normalizados pelo número de ataques $(\mathrm{N})$, e dados não normalizados (N). 


\section{Resultados}

As Tabelas 1 e 2 apresentam as médias dos indicadores técnicos; os coeficientes estruturais dos indicadores técnicos do campeonato todo e de cada temporada; e as taxas de acerto do modelo.Os resultados estão processados para os indicadores técnicos normalizados (Tabela 1) e para os indicadores técnicos não normalizados (Tabela 2). Para todos os jogos analisados com os indicadores técnicos normalizados pelo número de ataques (Tabela 1), os rebotes defensivos e assistências foram relevantes para discriminar as equipes vencedoras das perdedoras. Esses indicadores também foram relevantes em cada temporada analisada, apesar de alguns indicadores aparecerem discretamente em algumas temporadas (A2C em 08/09; A2E em 08/09, 11/12 e 12/13; A3C em 12/13). Já para todos os jogos analisados com os indicadores técnicos náo normalizados pelo número de ataques (Tabela 2), os rebotes defensivos, assistências e arremessos certos de três pontos foram relevantes para discriminar as equipes vencedoras das perdedoras. Esses indicadores também foram relevantes em quase todas as temporadas analisadas, com a adição de outros indicadores que aparecerem nas temporadas (A2E em 08/09, 11/12 e 12/13; A2C em 08/09, 10/11 e 12/13; A3C em 12/13, $13 / 14$ e $14 / 15)$.

Tabela 1. Descrição da análise discriminante com os indicadores técnicos normalizados.

\begin{tabular}{|c|c|c|c|c|c|c|c|c|c|}
\hline & & \multicolumn{8}{|c|}{ Temporadas } \\
\hline & & Todas & $08 / 09$ & $09 / 10$ & $10 / 11$ & $11 / 12$ & $12 / 13$ & $13 / 14$ & $14 / 15$ \\
\hline \multicolumn{2}{|c|}{ No de jogos } & 1912 & 236 & 221 & 252 & 252 & 350 & 316 & 285 \\
\hline IT & Média (DP) & \multicolumn{8}{|c|}{ Coeficientes Estruturais } \\
\hline $\mathrm{A} 3 \mathrm{C}$ & $6,0(2,3)$ & 0.29 & 0.19 & 0.23 & 0.23 & 0.22 & 0.31 & 0.29 & 0.29 \\
\hline A3E & $10,8(3,4)$ & -0.20 & -0.28 & -0.25 & -0.25 & -0.13 & -0.20 & -0.20 & -0.20 \\
\hline $\mathrm{A} 2 \mathrm{C}$ & $15,1(3,8)$ & 0.25 & 0.37 & 0.23 & 0.23 & 0.23 & 0.23 & 0.25 & 0.25 \\
\hline $\mathrm{A} 2 \mathrm{E}$ & $13,5(4,1)$ & -0.25 & -0.31 & -0.21 & -0.21 & -0.31 & -0.31 & -0.25 & -0.25 \\
\hline LLC & $11,7(4,8)$ & 0.22 & 0.25 & 0.22 & 0.23 & 0.19 & 0.17 & 0.22 & 0.22 \\
\hline LLE & $3,9(2,3)$ & -0.02 & 0.02 & -0.12 & -0.12 & 0.12 & 0.06 & -0.02 & -0.02 \\
\hline $\mathrm{RO}$ & $6,8(2,8)$ & 0.00 & -0.04 & -0.05 & -0.05 & 0.01 & -0.09 & 0.00 & 0.00 \\
\hline $\mathrm{RD}$ & $16,7(4,3)$ & 0.38 & 0.48 & 0.38 & 0.38 & 0.38 & 0.33 & 0.38 & 0.38 \\
\hline AS & $10,6(3,9)$ & 0.39 & 0.34 & 0.36 & 0.36 & 0.31 & 0.34 & 0.39 & 0.39 \\
\hline $\mathrm{BR}$ & $6,0(2,6)$ & 0.15 & 0.16 & 0.13 & 0.13 & 0.20 & 0.14 & 0.15 & 0.15 \\
\hline $\mathrm{TC}$ & $1,6(1,3)$ & 0.16 & 0.23 & 0.18 & 0.18 & 0.15 & 0.23 & 0.16 & 0.16 \\
\hline ER & $10,2(3,8)$ & -0.09 & -0.07 & -0.07 & -0.07 & -0.10 & -0.10 & -0.09 & -0.09 \\
\hline \multicolumn{2}{|c|}{ Eingenvalue } & 0.80 & 0.90 & 0.76 & 0.76 & 1.06 & 0.88 & 0.80 & 0.80 \\
\hline \multicolumn{2}{|c|}{ Correlação Canônica } & 0.67 & 0.69 & 0.66 & 0.66 & 0.72 & 0.68 & 0.67 & 0.67 \\
\hline \multicolumn{2}{|c|}{ Wilks' lambda } & 0.56 & 0.53 & 0.57 & 0.57 & 0.49 & 0.53 & 0.56 & 0.56 \\
\hline \multicolumn{2}{|c|}{ Significância } & 0.000 & 0.000 & 0.000 & 0.000 & 0.000 & 0.000 & 0.000 & 0.000 \\
\hline \multicolumn{2}{|c|}{ Taxa de acerto (\%) } & 81.8 & 82.8 & 80.1 & 80.1 & 84.1 & 81.6 & 81.8 & 81.8 \\
\hline
\end{tabular}

Notas. IT = indicadores técnicos; $\mathrm{A} 3 \mathrm{C}=$ arremessos de três pontos certos; $\mathrm{A} 3 \mathrm{E}=$ arremessos de três pontos errados; $\mathrm{A} 2 \mathrm{C}=$ arremessos de dois pontos certos; $\mathrm{A} 2 \mathrm{E}=$ arremessos de dois pontos errados; $\mathrm{LLC}=$ lances livres certos; $\mathrm{LLE}=$ lances livres errados; $\mathrm{RO}=$ rebotes ofensivos; $\mathrm{RD}=$ rebotes defensivos; $\mathrm{AS}=$ assistências; $\mathrm{BR}=$ bolas roubadas; $\mathrm{T}=$ tocos; $\mathrm{E}=$ erros. 
Tabela 2. Descrição da análise discriminante com os indicadores técnicos não normalizados.

\begin{tabular}{|c|c|c|c|c|c|c|c|c|c|}
\hline & & \multicolumn{8}{|c|}{ Temporadas } \\
\hline & & Todas & $08 / 09$ & $09 / 10$ & $10 / 11$ & $11 / 12$ & $12 / 13$ & $13 / 14$ & $14 / 15$ \\
\hline \multicolumn{2}{|c|}{ No de jogos } & 1912 & 236 & 221 & 252 & 252 & 350 & 316 & 285 \\
\hline IT & Média (DP) & \multicolumn{8}{|c|}{ Coeficientes Estruturais } \\
\hline A3C & $8.0(3.0)$ & 0.30 & 0.19 & 0.25 & 0.29 & 0.23 & 0.32 & 0.33 & 0.32 \\
\hline $\mathrm{A} 3 \mathrm{E}$ & $14.6(4.2)$ & -0.22 & -0.28 & -0.27 & -0.21 & -0.14 & -0.22 & -0.14 & -0.21 \\
\hline $\mathrm{A} 2 \mathrm{C}$ & $20.4(4.5)$ & 0.29 & 0.37 & 0.25 & 0.35 & 0.25 & 0.30 & 0.10 & 0.16 \\
\hline $\mathrm{A} 2 \mathrm{E}$ & $18.2(5.0)$ & -0.28 & -0.30 & -0.24 & -0.19 & -0.33 & -0.35 & -0.21 & -0.19 \\
\hline LLC & $15.7(6.0)$ & 0.23 & 0.26 & 0.25 & 0.22 & 0.21 & 0.19 & 0.27 & 0.17 \\
\hline LLE & $5.3(2.9)$ & -0.02 & 0.02 & -0.12 & -0.10 & 0.12 & 0.04 & -0.02 & -0.01 \\
\hline RO & $9.2(3.6)$ & 0.01 & -0.04 & -0.05 & 0.07 & 0.01 & -0.08 & 0.08 & 0.04 \\
\hline $\mathrm{RD}$ & $22.4(5.0)$ & 0.44 & 0.48 & 0.45 & 0.37 & 0.45 & 0.41 & 0.40 & 0.45 \\
\hline AS & $14.3(5.0)$ & 0.42 & 0.34 & 0.39 & 0.40 & 0.31 & 0.37 & 0.29 & 0.33 \\
\hline $\mathrm{BR}$ & $8.1(3.3)$ & 0.16 & 0.16 & 0.15 & 0.30 & 0.22 & 0.16 & 0.11 & 0.06 \\
\hline TC & $2.2(1.7)$ & 0.16 & 0.23 & 0.18 & 0.07 & 0.14 & 0.24 & 0.12 & 0.01 \\
\hline ER & $13.6(4.4)$ & -0.10 & -0.07 & -0.08 & -0.18 & -0.11 & -0.11 & -0.08 & -0.04 \\
\hline & envalue & 0.82 & 0.76 & 0.87 & 1,07 & 0.91 & 0.95 & 1,06 & 0.90 \\
\hline & áo Canônica & 0.67 & 0.66 & 0.68 & 0.72 & 0.69 & 0.70 & 0.72 & 0.69 \\
\hline & ' lambda & 0.55 & 0.57 & 0.53 & 0.48 & 0.52 & 0.51 & 0.49 & 0.53 \\
\hline & ficância & 0.000 & 0.000 & 0.000 & 0.000 & 0.000 & 0.000 & 0.000 & 0.000 \\
\hline & acerto (\%) & 81.9 & 79.6 & 84.3 & 84.1 & 81.6 & 83.9 & 85.1 & 82.8 \\
\hline
\end{tabular}

\section{Discussão}

A análise discriminante dos dados normalizados pelo número de ataques de todos os 1912 jogos do NBB apontou os indicadores técnicos assistências $(\mathrm{CE}=0.39)$ e rebotes defensivos $(\mathrm{CE}=0.38)$ como relevantes para diferenciar equipes vencedoras de perdedoras. As assistências aparecem como discriminantes também nas comparaçóes realizadas por Ibáñez et al., (2008, 2009) e García et al., (2013, 2014), exceto em jogos não balanceados da análise de García et al., (2014). As assistências podem indicar a importância do jogo coletivo ou a interação entre pelo menos dois jogadores durante o ataque, em detrimento dos ataques individuais. Os rebotes defensivos, que também discriminam vencedores de perdedores, indicam a necessidade de não proporcionar à equipe adversária uma nova chance após um ataque sem sucesso, que é atingido muitas vezes por uma postura defensiva forte (Sampaio, Ibáńez, Lorenzo \& Gómez, 2006). É certo considerar que permitir rebotes ofensivos pode aumentar a eficiência dos ataques da equipe adversária nos arremessos. As assistências e os rebotes ofensivos também foram discriminantes em todas as sete temporadas analisadas, apontando semelhanças entre elas.
Contudo, os arremessos de dois certos e errados e de três pontos certos também foram discriminantes em algumas temporadas. Cabe apontar que os arremessos de três pontos certos aparecem como discriminantes nas temporadas mais recentes (12/13, 13/14 e 14/15). Pode-se sugerir uma tendência atual na busca dos arremessos de três pontos pelas equipes de elite no Brasil, e sua alta eficiência pode ser um dos indicadores de vitória. Os arremessos de dois pontos errados aparecem como discriminantes também em três temporadas $(08 / 09,11 / 12$ e 12/13). Vale lembrar que este indicador não diferencia arremessos de meia distância de bandejas e, por isto, as médias das tentativas certas e erradas são maiores que dos arremessos de três pontos. Se os indicadores técnicos forem classificados como ofensivos (A3C, A3E, A2C, A2E, LLC, LLE, RO, AS e ER) e defensivos (RD, TC BR), apenas os rebotes defensivos aparecem como discriminante, tanto na análise de todos os jogos, quanto em todas as temporadas.

A comparação entre os resultados obtidos com indicadores técnicos não normalizados (Tabela 2) e normalizados (Tabela 1) aponta diferenças quanto ao conjunto de indicadores técnicos discriminantes para todos os jogos do NBB, pois além das assistências e rebotes defensivos também são discriminantes 
os arremessos de três pontos certos. Além disso, outros indicadores técnicos aparecem nas diferentes temporadas, todos ligados aos arremessos de dois e três pontos certos e/ou errados. Contudo, não foi verificada uma diferença substancial na taxa de acerto do modelo para todos os jogos entre os conjuntos normalizados e não normalizados, apontando que a normalização não melhorou e nem piorou estatisticamente a qualidade da análise discriminante entre equipes vencedoras e perdedoras.

Mesmo quando o tamanho da amostra de diferentes campeonatos, como a amostra de 3060 jogos analisados por Puente, Coso, Salinero \& Abián-Vicén, (2015) e a amostra de 1912 jogos do NBB, são analisadas pelos mesmos testes estatísticos nas mesmas condiçóes, é possível não encontrar os mesmos indicadores que discriminam vencedores e perdedores. Isso se deve a grande variabilidade de possibilidades que o basquetebol apresenta, o que indica que cada temporada ou campeonato sempre deverá ter características próprias.

\section{Conclusão}

Utilizando a análise discriminante foi possível encontrar os indicadores técnicos de jogo que separam as equipes vencedoras das perdedoras com uma taxa de acerto perto de $82 \%$ em sete temporadas do NBB, sem diferença substancial entre a análise com os indicadores técnicos normalizados e nâo normalizados.

Os indicadores técnicos que estão relacionados à vitória são os rebotes defensivos, sugerindo a importância de um forte trabalho defensivo nos arremessos adversários, e também as assistências, ressaltando a relevância de um trabalho coletivo no ataque e eficiência dos passes. Apesar da diferença entre o campeonato brasileiro do NBB e campeonatos espanhóis, os indicadores assistências e rebotes defensivos aparecem como discriminantes entre vencedores e perdedores nos dois contextos.

\section{Referências}

1. Almas, S.P. (2015). Análise das estatísticas relacionadas ao jogo que discriminam as equipes vencedoras das perdedoras no basquetebol profissional brasileiro Revista Brasileira Educação Física Esporte, 29, (4).

2. Barros, R.M.L., Misuta, M.S., Menezes, R.P., Figueroa, P.J., Moura, F.A., Cunha, S.A., Anido, R., \& Leite, N.J. (2007). Analysis of the distances covered by first division Brazilian soccer players obtained with an automatic tracking method. Journal of Sports Science and Medicine, 6 (2), 233-242.

3. Carvalho, A.B.C, \& Folle, A. (2014). Perfil estatístico dos atletas do NBB 2009/2010. Revista Mackenzie de Educação Física e Esporte, 13(1), 59-70.

4. Csataljay, G., James, N., Hughes, M., \& Dancs, H. (2012). Performance differences between winning and losing basketball teams during close, balanced and unbalanced quarters. Journal of Human Sport \& Exercise, 7, 356-364.

5. Fisher, R.A. (1936). The use of multiple measurements in taxonomic problems. Annals of Eugenics, ed.2, v. 7, n. 7, p. 179-188,

6. Galatti, L.R., Paes, R.R., Machado, G.V., \& Montero S.A. (2015). Campeonas del Mundo de Baloncesto: factores determinantes para el rendimiento de excelencia. Cuadernos de Psicología del Deporte, 15(3), 187-192.

7. García, J., Ibáñez, S.J., Gómez, M.A., \& Sampaio, J. (2014). Basketball Game-related statistics discriminating ACB league teams according to game location, game outcome and final score diferences. Int Journal of Performance Analysis in Sport, 14, 443-452.

8. García, J., Ibáńez, S.J., Santos, R.M., Leite, N., \& Sampaio, J. (2013). Identifying Basketball Performance Indicators in Regular Season and Playoff Games. Journal of Human Kinetics, 36, 161-168.

9. García, J., Sáez, J., Ibáñez, S.J., Parejo, I., \& Cañadas, M. (2009). Home advantage analysis in ACB league in season 2007-2008. Revista de Psicologia del Deporte, 18, 331-335.

10. Gómez, M.A., Lorenzo, A., \& Barakat, R. (2008a). Differences in game-related statistics of basketball performance by game location for men's winning and losing teams. Perceptual and Motor Skills, 106, 4350 .

11. Gómez, M.A., Lorenzo, A., Sampaio, J., Ibáñez, S.J., \& Ortega, E. (2008b). Game-Related Statistics that Discriminated Winning and
Losing Teams from the Spanish Men's Professional Basketball Teams. Coll. Antropol, 32(2), 451-456.

12. Ibáñez, S.J., García, J., Feu, S., Lorenzo, A., \& Sampaio, J. (2009). Effects of consecutive basketball games on the game-related statistics that discriminate winner and losing teams. 01 September 2009. Journal of Sports Science and Medicine 8, 458-462, de http://www.jssm.org.

13. Ibáñez, S.J., Sampaio, J., Feu, S., Lorenzo, A., Gómez, M.A, \& Ortega, E. (2008). Basketball game related statistics that discriminate between teams' season-long success. Europ. Journal of Sport Sciences, 8(6), 369-372.

14. Meneses, L.R., Junior, L.E.M.G., \& Almeida, M., B. (2016). Análise do desempenho do basquetebol brasileiro ao longo de três temporadasdo Novo Basquete Brasil. Revista Brasileira Ciências do Esporte, 38(1), 93-100.

15. Moreno, H.J. (1998) Fundamentos del deporte. Análisis de las estructuras del juego desportivo. Barcelona: Inde publicaciones, Capítulo 7, Los deportes de coperación/oposición. p. 119-137.

16. Norušis, M.J. (2004). SPSS 13.00 Advanced statistical procedures companion. Englewood-Cliffs, NJ: Prentice-Hall.

17. Oliver, D. (2004). Basketball on paper: Rules and tools for performance analysis. Washington, DC: Brassey's, Inc.

18. Puente, C., Coso, J.D., Salinero, J.J. \& Abián-Vicén, J. (2015). Basketball performance indicators during the ACB regular season from 2003 to 2013. International Journal of Performance Analysis in Sport, 15, 935-948.

19. Reis, M.R.S \& Sampaio, B.R. (2014) Diferenças de eficiência entre o novo basquete brasil, a national basketball assossiation (nba) e a euroleague: uma aplicação da análise envoltória de dados - dea. Área temática: Métodos Quantitativos.

20. Romarís, I. U., Refoyo, I., \& Lorenzo, J. (2016). Comparación de los ritmos de juego en Liga Femenina y ACB. Cuadernos de Psicología del Deporte, 16(2), 161-168.

21. Sampaio, J., \& Janeira, M. (2003). Statistical analyses of basketball team performance: understanding teams' wins and losses according to a different index of ball possessions. International Journal of Performance Analysis in Sport, 3(1), 40-49.

22. Sampaio, J., Ibáńez, S., Lorenzo, A., Gómez, M. (2006). Discriminative game-related statistics between basketball starters and nonstarters 
when related to team quality and game outcome. Perceptual and Motor Skills, 103(2), 486-494.

23. Tabachnick, B.G., \& Fidell, L.S. (2001). Using multivariate statistics. New York: Harper y Row Publishers.
24. Trninic, S., Dizdar, D., \& Luksic, E. (2002). Differences between winning and defeated top quality basketball teams in final of European club championship. Collegium Antropologicum. 26(2), 521-31. 